\title{
Disabling Language and the Nuances of Stigmatization
}

\author{
Andries Hiskes, Leiden University
}

\section{INTRODUCTION}

Illes and Lou's article concerning the language used in order to describe and nominate disabilities is both thoughtful and provocative. Presenting a survey of disability language employed across a vast range of cultures, Illes and Lou's study offers insight in how a term such as "mild developmental disability" might carry different meanings across cultures. Reflecting on these collected terms, Illes and Lou (2019) note: "Many questions arise from the database of disability terms we have assembled here. Among them, we ask: Given that language can be empowering or disabling, what place should cultural terminologies for disabilities have within disability conversations globally and in multicultural and pluralistic nations in the context of fostering an inclusive society?" (82).

This is a question that is as important as it is big. It is not only big in the sense that it would seem to require a complex answer (or seemingly, sets of answers), but also complex in that it is a question that in turn raises other questions: Would the term "inclusive society" itself mean the same across different cultures and groups of people? What would the striving toward such an inclusive society ask of various people in terms of how they speak of disability and their cultural adjustment? And, which I want to focus on in this commentary, how and to what extent can we speak of the disabling or empowering of language?

\section{STIGMATIZATION AND THE NUANCES OF DISABILITY TERMINOLOGY}

Discussing the categories in which disability terms are placed, Illes and Lou write that the term value "denotes the valence of the terms and was further subcategorized based on whether the term had elevating (positive) or stigmatizing (negative) connotations" (76). The term "stigma" was famously defined by Erving Goffman (1963) as follows:

While the stranger is present before us, evidence can arise of his possessing an attribute that makes him different from others in the category of persons available for him to be, and of a less desirable kind-in the extreme, a person who is quite thoroughly bad, or dangerous, or weak. He is thus reduced in our minds from a whole and usual person to a tainted, discounted one. Such an attribute is a stigma, especially when its discrediting effect is very extensive; sometimes it is also called a failing, a shortcoming, a handicap. (2-3)

Goffman's stigma is complex in that it is a recognized attribute by which someone is marked as "bad, dangerous, or weak," but that the cultural operation of recognizing something as a "negative" attribute is the stigma. As such, Goffman's definition offers insight into why a term such as "mental retardation" is experienced as being stigmatizing.

Yet by fully separating the stigmatizing from the elevating, we are also accepting it as an "either/or" construction, and are then bypassing the question whether the use of a term could potentially be both. When we use term "intellectual disability" in preference to "mental retardation," for example, does this mean that the first term is completely free of carrying stigmatizing qualities? As a term, "intellectual disability" might itself be perceived as being "politically correct," while actually still be experienced and/or used as a negative and stigmatizing attribute by certain groups in specific cultural contexts and situations, even if the nature of the stigma may be different, in some respects, from the one attached to "mental retardation."

I am not arguing for a kind of banal relativism such that the language used to talk about disability ultimately would not matter, as I believe it does. Rather, I am arguing for a heightened and continuous sense of awareness of the fallacy of the bifurcation that certain terms would be stigmatizing and disabling, while others would be elevating and empowering. Second, I want to argue that we should examine and consider how terms are stigmatizing and empowering (and to what extent) in the cultural contexts in which they are used. 
If we don't strive to understand the interests of the various involved parties that are involved in the process of stigmatization, studies like that of Illes and Lou risk becoming reduced to "checklists" for which words to use, without the accompanying history of how these words have traveled and developed throughout different cultural contexts. Although Illes and Lou use the term "stakeholders" in their study, it seems that they use these terms mainly to refer to those people that are identified with having disabilities, which would in turn ignore that there are nearly always more "stakeholders" involved in the cultural processes that underlie stigmatization.

In their study Narrative Prosthesis (2000), David Mitchell and Sharon Snyder have argued that the stigmatization of disability is often a rhetorical device that propels forward narratives in which disability is featured, hence the title. They show how in various narratives, individuals and groups often have vested interests in people with disabilities and their places in society. Through this, they offer a valuable insight in the process of stigmatization: that the underlying motivation to stigmatize often involves the distribution of things such as power, wealth, and access to resources and education. Tobin Siebers, meanwhile, has argued in his Disability Theory (2008) that reclaiming and redefining the language around disability by disabled people may offer new possibilities to engage in identity politics and employ the language concerning disability on their own terms. By taking the language around disability in new directions for disabled people, Siebers' study suggests that this might allow us to reframe the context and values that become attached to the language around disability.

The motivation to name these books in commenting on Illes and Lou's study is because I believe in the added value of placing academic discourse concerning bioethics and neuroethics in conversation with that of cultural disability studies. Although Illes and Lou acknowledge that the terminology surrounding disability is nuanced and varied, by dividing the collected terms into a schema, one needs to critically consider to what extent such a division primarily serves the purpose of offering a comprehensive overview and to what extent such a schematic presentation could concurrently do justice to the complexity of the sociocultural practices in which they are used. If more extensive anthropological fieldwork were done in tandem, in adjacency to reading these results in the contexts of the theory produced by cultural disability studies, a richer interpretation could be offered that would delineate the important nuances of disability terminology that now only remain briefly mentioned.

\section{DISABLING LANGUAGE AND FUTURE PROSPECTS}

Next to the term "stigmatizing," Illes and Lou also employ the term "disabling" in their study on disability terminology. Although the two are not explicitly differentiated in the study, I believe it relevant to understand their potential differences and their value in studying disability terminology. As was noted in the Goffman quote, stigma rests on the operation of recognizing and perceiving a certain attribute in order to be able to differentiate a person as weak, bad, or dangerous. But this process of assigning the stigma does not yet fully cover the myriad ways in which language relates to the practice of stigmatization. Although it is of course fully possible to stigmatize someone by calling that person "retarded" in a given cultural context, language can also be disabling in other ways, even when not referring to disability explicitly. We can, for instance, easily imagine ways in which language is disabling while only implicitly referring to the stigma attached: for example, "Don't give that book to him, it's much too complex!" This example sentence does not nominate or describe a disability directly, but it is disabling by limiting the behavior a person should engage in. By circumventing the explicit reference to disability, such sentences may be harder to interpret as being stigmatizing but are not any less so.

Though such language usage falls outside the scope of Illes and Lou's article, as they situate their study in the context of "fostering a more inclusive society" it is important to recognize that the terminology used to describe and nominate disability is only a part of the stigmatizing practices attached to it. As political sensibilities to disability terminology may increase over time, it would arguably be somewhat naive to think that stigmatizing practices would concurrently lessen, dissolve, or disappear. Instead, as terminology surrounding disability becomes more nuanced across various cultural contexts, so too may the accompanying processes of stigmatization, and it may become increasingly difficult to recognize them as such.

This relationship between stigmatizing and disabling language also offers perspective on the differentiation between descriptive categories and nominative terms in Illes and Lou's study. In the schema provided, the nominative terms 
are separated from descriptive categories, but thereby any relationship between the nominative and the stigmatizing cannot be established. Referring back to my earlier argument where I wondered if certain terms can be entirely free of stigma, I also question whether nominative terms are entirely free of stigma and can thereby remain detached from the rest of the presented schema.

If we momentarily ignore the nuances of stigmatization I've mentioned, Illes and Lou's study offers important insight into the sheer difference in number of terms that are stigmatizing and elevating. Their study finds that $46 \%$ of the surveyed terms are dominantly stigmatizing, 39\% are medicalizing, and only 14\% are considered "elevating." Similarly to how "elevating terms" might still carry stigma to some extent, the same question could of course be raised for those terms considered "medicalizing." That said, the percentages given clearly show how elevating terms are in the minority. These numbers initially do not appear to offer much to rejoice about, but as Illes and Lou suggest in their study, they do offer the questions of what cross-cultural dialogues have to offer. In future study, I think it would be worthwhile to explore how the terms that were classified as being "elevating" operate in their cultural contexts, and consequently to consider whether and how those terms, or the concepts underlying them, could be relevant to other cultures and groups. Such future study however, should not be the task of bioethics and neuroethics alone; a fruitful collaboration and interchange with cultural disability studies and anthropology would be sure to enrich the interpretation and potential usage of future disability terminology.

\section{REFERENCES}

Goffman, E. 1963. Stigma; notes on the management of spoiled identity. Englewood Cliffs, NJ: Prentice-Hall.

Illes, J., and H. Lou. 2019. A cross-cultural neuroethics view on the language of disability. American Journal of Bioethics: Neuroscience 10(2): 75-84.

Mitchell, D., and S. Snyder. 2000. Narrative prosthesis: Disability and the dependencies of discourse. Ann Arbor, MI: University of Michigan Press.

Siebers, T. A. 2008. Disability theory. Ann Arbor, MI: University of Michigan Press. 